\title{
Harvest of Product from Cell Media
}

National Cancer Institute

\section{Source}

National Cancer Institute. Harvest of Product from Cell Media. NCI Thesaurus. Code C112936.

Collection of a material of interest from a cell culture supernatant. 